\title{
Energy Levels, Lifetimes, and Transition Probabilities for Rb(XXIII)
}

\author{
Min Xu ${ }^{a}$, Feng $\mathrm{Hu}^{b, *}, \mathrm{Yan} \mathrm{Sun}^{b}$, Yuewu Pan ${ }^{b}$ And Hao $\mathrm{LiU}^{c}$ \\ ${ }^{a}$ Key Laboratory of State Ethnic Affairs Commission for Electronic \\ and Information Engineering, Southwest Minzu University, \\ Chengdu 610041, People's Republic of China \\ ${ }^{b}$ School of Physics and New Energy, Xuzhou University of Technology, \\ Xuzhou 221018, People's Republic of China \\ ${ }^{c}$ Research Center of Laser Fusion, China Academy of Engineering Physics, \\ Mianyang 621900, People's Republic of China
}

Received: 31.01.2021 \& Accepted: 09.06.2021

Doi: $10.12693 /$ APhysPolA.140.50

*e-mail: hufengscu@139.com

\begin{abstract}
Energy levels, radiative rates, lifetimes, hyperfine structures and Landé $g_{J}$-factors for electric-dipole (E1), electric-quadrupole (E2), magnetic-dipole (M1), and magnetic-quadrupole (M2) transitions among the lowest 41 levels belonging to the $n=3$ states $\left(1 s^{2} 2 s^{2} 2 p^{6}\right) 3 s^{2} 3 p^{3}, 3 s 3 p^{4}$, and $3 s^{2} 3 p^{2} 3 d$ in $\mathrm{Rb}(\mathrm{XXIII})$ are calculated through the multiconfiguration Dirac-Hartree-Fock method. High-accuracy calculations have been performed as benchmarks in the request for accurate treatments of relativity, electron correlation, and quantum electrodynamic effects in multi-valence-electron systems. The calculated energy levels are in excellent agreement with the experimental results and the theoretical ones, wherever available. The calculated values including core-valence correction are found to be in good agreement with other theoretical and experimental values. The present results are reported as benchmarks for future calculations and measurements.
\end{abstract}

topics: energy levels, radiative rates, transition probabilities

\section{Introduction}

The most frequent use of $\mathrm{Rb}$ is in the field of astrophysics and atomic physics. The formation of the rubidium resonance lines has been used to investigate the temperature and abundance of elements in cool stars [1]. Rubidium is a key element for the slow neutron-capture mechanismsprocess diagnostic [2]. The absorption spectroscopy of rubidium was performed to improve the accuracy of the atomic clock [3]. Rubidium vapor was pumped to study proton polarization produced in the process of polarized electron capture [4]. Experimental spectra are mainly from low ionized $\mathrm{Rb}$ ions, and there were no experimental results for $\mathrm{Rb}(\mathrm{XXI})-\mathrm{Rb}$ (XXXVII) (except four ions) [5]. The energy levels and transition probabilities of $\mathrm{Rb}(\mathrm{XXI})-\mathrm{Rb}(\mathrm{XXXVII})$ quoted by the Atomic Spectra Database of the National Institute of Standards and Technology (NIST ASD) were obtained from the predictions of isoelectronic sequences [6]. For Rb(XXIII), no measurements of the spectrum have been made, and the study was mainly from phosphorus isoelectronic sequence. By investigating magnetic dipole transition within the $3 s^{2} 3 p^{3}$ ground state, the ground state levels, wavelengths, and transition probabilities were predicted by Sugar and Kaufman [7]. Later, four levels in the $3 s^{2} 3 p^{2} 3 d$ configuration and wavelengths for transitions to those levels from the ground term were made by Sugar et al. [8]. The multiconfiguration Dirac-Fock technique was used to calculate the transition rates for $\mathrm{Rb}$ (XXIII) by Huang [9].

Träbert conducted a critical assessment of theoretical calculations of structure and transition probabilities from an experimenter's perspective [10]. He pointed out that new computations can match the measurement, fill gaps and suggest revisions closely with almost spectroscopic accuracy. These citations of theoretical work as well as the ones for experimental data are certainly incomplete. The citations concerned several P-like ions calculations, and the trend attracted attention. Consequently, limited energy levels or transitions were considered, or selected configurations were discussed [7, 8] and some results were given in a graphic form [9]. There still exist some problems, such as the identification of terms and strong mixing of configurations, which will be discussed in detail in the next part of this paper.

In this paper, the MCDHF method is performed to calculate E1, E2, M1, and M2 wavelengths, oscillator strengths, transition probabilities, and finestructure levels for $\mathrm{Rb}$ (XXIII) using the new release 
of the GRASP2K code $[11,12]$ and the fully relativistic flexible atomic code (FAC) program [13], which was used to check the energy levels. Configurations $\left(1 s^{2} 2 s^{2} 2 p^{6}\right) 3 s^{2} 3 p^{3}, 3 s 3 p^{4}$, and $3 s^{2} 3 p^{2} 3 d$ are reported in this calculation. Based on our previous work $[14,15]$, in this paper, the valence-valence (VV) and core--valence (CV) correlation effects are considered systematically. The Breit interactions (BI) and quantum electrodynamics (QED) effects have been added. This computational approach enables us to present a consistent and improved data set of all-important E1, E2, M1, and M2 transitions of the $\mathrm{Rb}$ (XXIII) spectra, which are useful for identifying transition lines in further investigations.

\section{Theoretical methods}

\subsection{MCDHF method}

The multiconfiguration Dirac-Hartree-Fock (MCDHF) method has recently been described in great detail by Grant [16]. Hence, we only repeat its essential features here. We start from the Dirac-Coulomb Hamiltonian

$H_{\mathrm{DC}}=\sum_{i=1}^{N}\left(c \boldsymbol{\alpha}_{i} \cdot \boldsymbol{p}_{i}+\left(\beta_{i}-1\right) c^{2}+V_{i}^{N}\right)+\sum_{i>j}^{N} \frac{1}{r_{i j}}$.

Here, $V^{N}$ is the monopole part of the electronnucleus Coulomb interaction, $\alpha$ and $\beta$ are the $4 \times 4$ Dirac matrices, and $c$ is the speed of light in atomic units. The atomic state functions (ASFs) describing different fine-structure states are obtained as linear combinations of symmetry-adapted configuration state functions (CSFs):

$$
\left|\gamma J M_{J}\right\rangle=\sum_{j=1}^{N_{C S F s}} c_{j}\left|\gamma_{j} J M_{J}\right\rangle
$$

where $J$ and $M$ are the angular quantum numbers and $P$ is the parity. Parameter $\gamma_{i}$ denotes another appropriate labeling of the configuration state function $i$, for example, orbital occupancy, and coupling scheme. Normally, the label $\gamma$ is the same as the label of the dominating CSF. The CSFs are built from products of one-electron Dirac orbitals. In the relativistic self-consistent field (RSCF) procedure, both the radial parts of the Dirac orbitals and the expansion coefficients are optimized to self-consistency. The Breit interaction

$$
\begin{aligned}
& H_{\text {Breit }}=-\sum_{i<j}^{N}\left[\boldsymbol{\alpha}_{i} \cdot \boldsymbol{\alpha}_{j} \frac{\cos \left(\omega_{i j} r_{i j} / c\right)}{r_{i j}}\right. \\
& \left.+\left(\boldsymbol{\alpha}_{i} \cdot \nabla_{i}\right)\left(\boldsymbol{\alpha}_{j} \cdot \nabla_{j}\right) \frac{\cos \left(\omega_{i j} r_{i j} / c\right)-1}{\omega_{i j}^{2} r_{i j} / c^{2}}\right]
\end{aligned}
$$

was included in the Hamiltonian. The photofrequencies $\omega_{i j}$, used for calculating the matrix elements of the transverse photo-interaction, were taken as the difference of the diagonal Lagrange multipliers associated with the Dirac orbitals.
In the relativistic configuration interaction (RCI) calculation, leading QED corrections [17], selfinteraction, and vacuum polarization were also included.

\subsection{MBPT method}

In the MBPT method implemented in the FAC package [13], the Dirac-Coulomb-Breit Hamiltonian can be written as

$$
H_{\mathrm{DCB}}=\sum_{i}^{N}\left(h_{d}(i)-\frac{Z}{r_{i}}\right)+\sum_{i<j}^{N}\left(\frac{1}{r_{i j}}+B_{i j}\right),
$$

where $h_{d}$ is the free-electron Dirac Hamiltonian, $r_{i}$ is the radial coordinate of the electron $i, r_{i j}$ is the distance between the electrons $i$ and $j$, and $Z$ is the nuclear charge number. The frequency-independent Breit interaction is denoted as $B_{i j}$.

The $H_{D C B}$ is split up into a model Hamiltonian $H_{0}$ and a perturbation $V$, namely

$$
\begin{aligned}
& H_{0}=\sum_{i} h_{d}(i)+U\left(r_{i}\right), \\
& V=-\sum_{i}\left(\frac{Z}{r_{i}}+U\left(r_{i}\right)\right)+\sum_{i<j}^{N}\left(\frac{1}{r_{i j}}+B_{i j}\right) .
\end{aligned}
$$

Here, U(r) is approximated by a local central potential and derived from an RSCF calculation. The eigenfunctions of $H_{0}$ are divided into a model space $M$ and an orthogonal space $N$. A non-Hermitian effective Hamiltonian whose eigenvalues are the true eigenenergies of the full Hamiltonian can be constructed in the $M$ space. By solving the generalized eigenvalue problem for the first-order effective Hamiltonian, we can obtain the eigenvalues in the second order. With this method, the CI effects within the $M$ space are included for all orders, while the interaction between $M$ and $N$ is taken into account for the second order. Finally, several small corrections to the Hamiltonian, such as QED, are also included.

\subsection{Calculation procedure}

The $3 s^{2} 3 p^{3}, 3 s 3 p^{4}$, and $3 s^{2} 3 p^{2} 3 d$ configurations define the multireference (MR) for the even and odd parities, respectively. As a starting point, MCDHF calculations in the EOL scheme were performed for even and odd states using configuration expansions including all lower states of the same $J$ symmetry and parity, and a Dirac-Coulomb version was used, for the optimization of the orbitals, including BI in a final configuration interaction calculation [16].

The calculations for the even states were based on CSF expansions obtained by allowing single (S) and double (D) substitutions of orbitals in the even MR configurations to an increasingly active set of orbitals. Similarly, the calculations for the odd states were based on CSF expansions obtained by allowing $\mathrm{S}$ and D substitutions of orbitals in the odd MR configurations to an increasingly active set of orbitals. A similar calculation procedure has been introduced 
in a previous paper [14], thus in the current paper we only give an outline. For P-like ions, the ground and first excited configurations are $3 s^{2} 3 p^{3}$, and $3 s^{2} 3 p^{2} 3 d$, respectively. In the first step, the active set $(\mathrm{AS})$ is

$$
\mathrm{AS} 1=\{3 s, 3 p, 3 d\} .
$$

Then, we increase the active set in the following way:

$$
\begin{aligned}
& \mathrm{AS} 2=\mathrm{AS} 1+\{4 s, 4 p, 4 d, 4 f\}, \\
& \mathrm{AS} 3=\mathrm{AS} 2+\{5 s, 5 p, 5 d, 5 f, 5 g\}, \\
& \mathrm{AS} 4=\mathrm{AS} 3+\{6 s, 6 p, 6 d, 6 f, 6 g\}, \\
& \mathrm{AS} 5=\mathrm{AS} 4+\{7 s, 7 p, 7 d, 7 f, 7 g\} .
\end{aligned}
$$

In our $\mathrm{VV}$ method, we set $1 s^{2} 2 s^{2} 2 p^{6}$ as our core electrons in the calculation. Then, we considered increasing the principal quantum number $n$ and optimized the orbitals AS1, AS2, AS3, AS4, and AS5.

In the $\mathrm{CV}$ model, we set $1 s^{2} 2 s^{2} 2 p^{5}$ as our core electrons, then we optimized the layer by $n$. We generated the CSFs of the form of $1 s^{2} 2 s^{2} 2 p^{5} \mathrm{ASn}$, $n=1-4$. Also, the CSFs of CV have the form of $1 s^{2} 2 s^{1} 2 p^{6}$ ASn, $n=1-4$.

In our MBPT calculation, the $3 l^{5}, 43 l^{4} 4 l$, and $3 l^{4} 5 l$ configurations are contained in the model space $M$, and all possible configurations that are generated by SD excitations from the $M$ space are contained in space $N$.

All calculations were done in $j j$-coupling. To give a good consistency with the labeling system, we used the NIST ASD and other sources. The GRASP2K procedure JJ2LSJ [18] was used for the transformation of ASFs from a $j j$-coupled CSF basis into an $L S J$-coupled CSF basis for the results.

\section{Results and discussion}

Results for $\left(1 s^{2} 2 s^{2} 2 p^{6}\right) 3 s^{2} 3 p^{3}, \quad 3 s 3 p^{4}, \quad$ and $3 s^{2} 3 p^{2} 3 d$ configurations of $\mathrm{Rb}$ (XXIII) give rise to the low-lying 41 levels listed in Table I, where we compared our results with the experimental data compiled by NIST [6]. The NIST database listed the energies for the 12 out of the present 41 excitedlevels in $\mathrm{Rb}(\mathrm{XXIII})$. The average values of the difference for MCDHFCV energies of the 41 levels are

- $\left(\frac{|\mathrm{MR}-(n=4)|}{\max (\mathrm{MR}, n=4)}\right)$,

- $0.164 \%\left(\frac{|(n=5)-(n=4)|}{\max (n=5, n=4)}\right)$,

- $0.019 \%\left(\frac{|(n=6)-(n=5)|}{\max (n=6, n=5)}\right)$,

- $\left.0.005 \%\left(\frac{|(n=7)-(n=6)|}{\max (n=7, n=6)}\right)\right)$.

The principal number was limited to $n=7$. For the VV calculation, it is not very difficult to get convergence for a higher principal number $(n \geq 8)$, but for the CV calculation, the convergence is difficult. The number of CSFs would increase very rapidly when we include the $n \geq 8$ orbitals, and it is hard to get convergence. Also, because of the computer calculation limit and the problem of the program

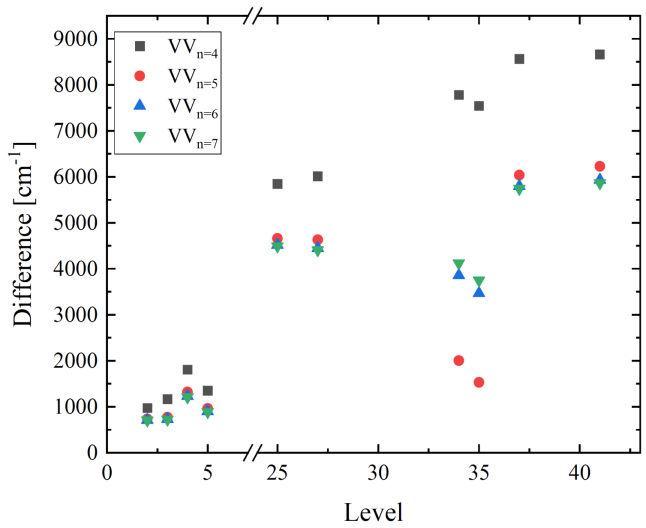

Fig. 1. Energy difference between the valencevalence correlation results and the energies for the 12 out of the lowest 41 levels from NIST.

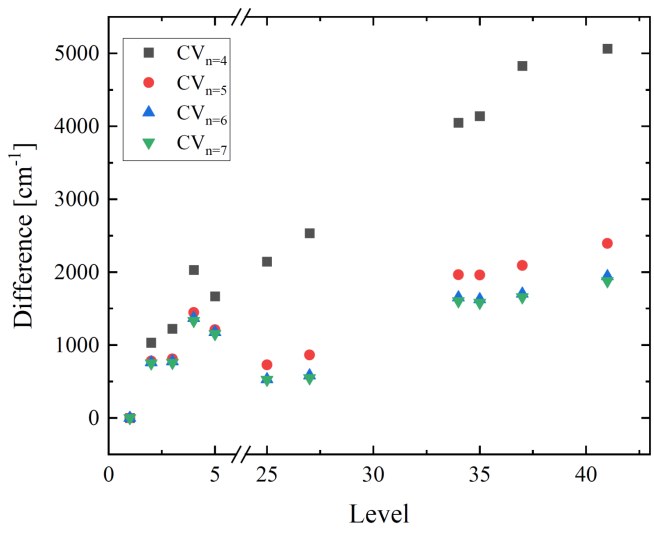

Fig. 2. Description as in Fig. 1, but for the corevalence correlation results.

GRASP2K code itself, we only compare the VV and $\mathrm{CV}$ models on an equal footing, as mentioned above.

Figure 1 shows the mean values (with the standard deviation) of the relative differences between $\mathrm{VV}_{n}$ and NIST are 881 and $4728 \mathrm{~cm}^{-1}$. The smallest difference is $750 \mathrm{~cm}^{-1}$ lower than NIST $\left(3 s^{2} 3 p^{3}{ }^{2} D_{3 / 2}\right)$, and the biggest difference can be up to $8660 \mathrm{~cm}^{-1}\left(3 s^{2} 3 p^{2} 3 d\left({ }^{3} P\right){ }^{2} F_{5 / 2}\right)$. Figure 2 shows the mean values (with the standard deviation) of the relative differences between $\mathrm{CV}_{n}$ and NIST are 997 and $1297 \mathrm{~cm}^{-1}$. This can be treated as a good example of calculations with the necessary correlations included. As can be seen from Figs. 1 and 2, some results considering more configurations are not better than those with fewer configurations. This can be due to configuration mixing. The present results in Fig. 3 are the VV and CV calculations with $n=7$. For $3 s^{2} 3 p^{3}$, the VV results agree well with NIST in the range of $0.36 \%$ to $0.81 \%$, while the CV results - in the range of $0.47 \%$ to $0.87 \%$. For $3 s^{2} 3 p^{2} 3 d$, the VV results agree well with NIST in the range of $0.36 \%$ to $0.53 \%$, while the CV results - in the range of $0.06 \%$ to $0.16 \%$. Theoretical results from FAC differ from NIST in the range of $-0.62 \%$ to $7.46 \%$ for $3 s^{2} 3 p^{3}$ and $-0.01 \%$ to $1.07 \%$ for $3 s^{2} 3 p^{2} 3 d$. 
Energies (in $\left[\mathrm{cm}^{-1}\right]$ ) relative to the ground state for the lowest 41 levels in $\mathrm{Rb}$ (XXIII).

TABLE I

\begin{tabular}{|c|c|c|c|c|c|c|c|c|c|c|}
\hline Key & Configuration & $\mathrm{V}_{n=4}$ & $\mathrm{~V}_{n=5}$ & $\mathrm{VV}_{n=6}$ & $\mathrm{~V}_{n=7}$ & $\mathrm{CV}_{n=4}$ & $\mathrm{CV}_{n=5}$ & $\mathrm{CV}_{n=6}$ & $\mathrm{CV}_{n=7}$ & Exp. \\
\hline 1 & ${ }^{2} 3 p^{3}{ }^{4} S_{3 / 2}$ & - & 0 & 0 & 0 & 0 & 0 & 0 & 0 & 0 \\
\hline 2 & ${ }^{2} 3 p^{3}{ }^{2} D_{3 / 2}$ & 52 & 87213 & 88 & 7182 & 09 & 7258 & 87240 & 87228 & 86478 \\
\hline 3 & ${ }^{2} 3 p^{3}{ }^{2} D_{5 / 2}$ & - & 11 & 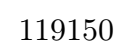 & 0 & 119642 & 119228 & 119193 & 119174 & 18418 \\
\hline 4 & ${ }^{2} 3 p^{3}{ }^{2} P_{1 / 2}$ & 168148 & 1 & 167572 & 1 & 0 & 8 & 167711 & 167674 & 166341 \\
\hline 5 & $s^{2} 3 p^{3}{ }^{2} P_{3 / 2}$ & 2 & 2422 & 1 & 24 & 2429 & 9 & 242435 & 242411 & 41259 \\
\hline 6 & $53 p^{4}{ }^{4} P_{5 / 2}$ & & 543 & 5 & 5 & 544381 & 55 & 544137 & 544126 & \\
\hline 7 & $s 3 p^{4}{ }^{4} P_{3 / 2}$ & & 601 & & 6 & 6 & 7 & 5 & 60 & \\
\hline 8 & ${ }_{s} 3 p^{4}{ }^{4} P_{1 / 2}$ & 8 & 6145 & 1 & 61 & 6152 & 614831 & 96 & 614877 & \\
\hline 9 & $s 3 p^{42} D_{3 / 2}$ & 677795 & 677 & 677590 & 677558 & 6780 & 0 & 677524 & 7 & \\
\hline 10 & $3 p^{42} D_{5 / 2}$ & 707891 & 707783 & 707654 & 707620 & 708 & 3 & 49 & 24 & \\
\hline 11 & ${ }^{2} 3 p^{2} 3 d\left({ }^{3} P\right.$ & 749880 & 749327 & 749186 & 749153 & 749316 & 748321 & 748237 & 748242 & \\
\hline 12 & ${ }^{2} 3 p^{2} 3 d\left({ }^{3} P\right)^{2} P_{3 / 2}$ & 768766 & 768609 & 768496 & 768469 & 768151 & 767628 & 767615 & 767628 & \\
\hline 13 & $3 p^{42} S_{1 / 2}$ & 772759 & 772463 & 772275 & 772230 & 772218 & 771394 & 771301 & 771265 & \\
\hline 14 & ${ }^{2} 3 p^{2} 3 d\left({ }^{3} P\right)^{4} F_{5 / 2}$ & 778528 & 777999 & 777883 & 777857 & 777809 & 776901 & 776855 & 776885 & \\
\hline 15 & ${ }^{2} 3 p^{2} 3 d\left({ }^{1} D\right)^{2} F_{5 / 2}$ & 813237 & 812654 & 812534 & 812506 & 812619 & 811707 & 811649 & 811675 & \\
\hline 16 & ${ }^{2} 3 p^{2} 3 d\left({ }^{3} P\right)^{4} F_{7 / 2}$ & 825405 & 825000 & 824909 & 824889 & 824867 & 824115 & 824107 & 824149 & \\
\hline 17 & ${ }^{2} 3 p^{2} 3 d\left({ }^{3} P\right)^{4} D_{1 / 2}$ & 830540 & 830140 & 830001 & 829970 & 829994 & 829085 & 829050 & 829063 & \\
\hline 18 & ${ }^{2} 3 p^{2} 3 d\left({ }^{3} P\right)^{4} D_{7 / 2}$ & 838508 & 837946 & 837828 & 837801 & 837960 & 837039 & 837004 & 837036 & \\
\hline 19 & ${ }^{2} 3 p^{2} 3 d\left({ }^{3} P\right)^{4} D_{3 / 2}$ & 847122 & 731 & 846623 & 846600 & 846226 & 845405 & 845393 & 845425 & \\
\hline 20 & ${ }^{2} 3 p^{2} 3 d\left({ }^{3} P\right)^{4} F_{9 / 2}$ & 957 & 43 & 858231 & 858205 & 858497 & 857532 & 857496 & 32 & \\
\hline 21 & ${ }^{2} 3 p^{2} 3 d\left(^{3}\right.$ & 619 & 02 & 996 & 0973 & 870786 & 869912 & 869885 & 869921 & \\
\hline 22 & ${ }^{2} 3 p^{2} 3 d\left({ }^{3} P\right)^{2} P_{1 / 2}$ & 877 & 2 & 39 & 48 & 878572 & 69 & 877693 & 674 & \\
\hline 23 & ${ }^{2} 3 p^{2} 3 d\left({ }^{1} D\right.$ & 922925 & 9 & 9 & 69 & 922 & 80 & 920773 & 87 & \\
\hline 24 & $3 p^{42} P_{3 / 2}$ & & & & & & & 931159 & & \\
\hline 25 & ${ }^{2} 3 p^{2} 3 d\left({ }^{3} P\right.$ & 94 & . & & 21 & 72 & 58 & 940760 & 940754 & 940230 \\
\hline 26 & ${ }^{2} 3 p^{2} 3 d\left({ }^{1} D\right.$ & 95 & 952 & 95 & 95 & 95 & 17 & 952031 & 952049 & \\
\hline 27 & $3 p^{2} 3 d\left({ }^{3} P\right)^{4} P_{3 / 2}$ & 960392 & 959010 & 958828 & 958786 & 956915 & 955243 & 954961 & 954926 & 954380 \\
\hline 28 & ${ }^{2} 3 p^{2} 3 d\left({ }^{3} P\right)^{4} P_{1 / 2}$ & 966938 & 965 & 96 & 99 & 96 & 10 & 961335 & 961305 & \\
\hline 29 & ${ }^{2} 3 p^{2} 3 d\left({ }^{1} D\right)^{2} G_{9 / 2}$ & 977808 & 976467 & 97 & 976232 & 977488 & 721 & 975582 & 975587 & \\
\hline 30 & ${ }^{2} 3 p^{2} 3 d\left({ }^{1} S\right)^{2} D_{3 / 2}$ & 986026 & 984227 & 983922 & 983850 & 984567 & 982400 & 982013 & 981958 & \\
\hline 31 & $3 s^{2} 3 p^{2} 3 d\left({ }^{3} P\right)^{2} D_{5 / 2}$ & 1019986 & 1017896 & 017630 & 1017569 & 1016460 & 1014099 & 1013682 & 1013618 & \\
\hline 32 & $3 s 3 p^{4}{ }^{2} P_{1 / 2}$ & 1020606 & 1019476 & 1019274 & 1019229 & 1016727 & 244 & 1015042 & 1015003 & \\
\hline 33 & ${ }^{2} 3 p^{2} 3 d\left({ }^{1} D\right)^{2} P_{1 / 2}$ & 1050214 & 1047897 & 1047584 & 1047503 & 1047188 & 1044513 & 1044008 & 1043917 & \\
\hline 34 & ${ }^{2} 3 p^{2} 3 d\left({ }^{1} D\right)^{2} D_{3 / 2}$ & 1051482 & 1049750 & 1049523 & 1049471 & 1047748 & 1045665 & 1045352 & 1045303 & 1043700 \\
\hline 35 & ${ }^{2} 3 p^{2} 3 d\left({ }^{1} D\right)^{2} D_{5 / 2}$ & 1055744 & 1053870 & 1053628 & 1053574 & 1052339 & 1050160 & 1049826 & 1049780 & 1048200 \\
\hline 36 & ${ }^{2} 3 p^{2} 3 d\left({ }^{1} S\right)^{2} D_{5 / 2}$ & 1076234 & 1074206 & 1073878 & 1073794 & 1075349 & 1072876 & 1072506 & 1072453 & \\
\hline 37 & ${ }^{2} 3 p^{2} 3 d\left({ }^{3} P\right)^{2} F_{7 / 2}$ & 1092645 & 1090117 & 1089876 & 1089821 & 1088907 & 1086172 & 1085782 & 1085737 & 1084080 \\
\hline 38 & ${ }^{2} 3 p^{2} 3 d\left({ }^{1} D\right)^{2} P_{3 / 2}$ & 1110640 & 1108421 & 1108121 & 1108048 & 1107458 & 1104918 & 1104436 & 1104351 & \\
\hline 39 & ${ }^{2} 3 p^{2} 3 d\left({ }^{1} D\right)^{2} S_{1 / 2}$ & 1124309 & 1122297 & 1122001 & 1121930 & 1120720 & 1118361 & 1117994 & 1117924 & \\
\hline 40 & ${ }^{2} 3 p^{2} 3 d\left({ }^{3} P\right)^{2} D_{3 / 2}$ & 1155429 & 1153084 & 1152764 & 1152686 & 1152336 & 1149651 & 1149177 & 1149102 & \\
\hline 41 & $3 s^{2} 3 p^{2} 3 d\left({ }^{3} P\right)^{2} F_{5 / 2}$ & 1163080 & 1160648 & 1160353 & 1160286 & 1159484 & 1156814 & 1156362 & 1156297 & 1154420 \\
\hline
\end{tabular}

To see the BI and QED effects more clearly, the contributions to the MCDHF excited energies of the 40 fine-structure levels of $\mathrm{Rb}(\mathrm{XXIII})$ in $\mathrm{cm}^{-1}$ and percentage are shown in Fig. 4a and $\mathrm{b}$, respectively. The inspection of Fig. 4 reveals that the BI and QED corrections are significant, generally lowering the excited levels by the mean value of $0.61 \%$. As can be seen, the contribution of the Breit interaction is about $-1.47 \%-0.29 \%$ $\left(-1956 \mathrm{~cm}^{-1}-290 \mathrm{~cm}^{-1}\right)$, and the contribution of QED is $-0.49 \%-0.127 \%\left(-2692 \mathrm{~cm}^{-1}-281 \mathrm{~cm}^{-1}\right)$.

The Dirac-Fock wave functions with a minimum number of radial functions are not sufficient to represent the occupied orbitals. Extra 


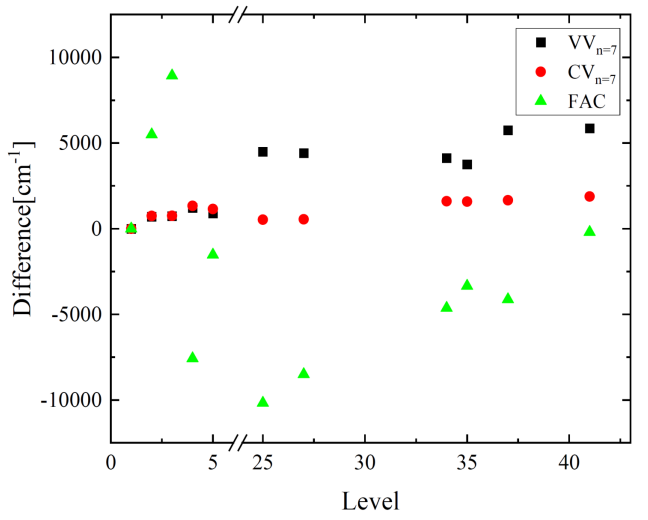

Fig. 3. Differences (in $\left[\mathrm{cm}^{-1}\right]$ ) of various theoretical energies from the NIST-compiled values in $\mathrm{Rb}(\mathrm{XXIII})$.
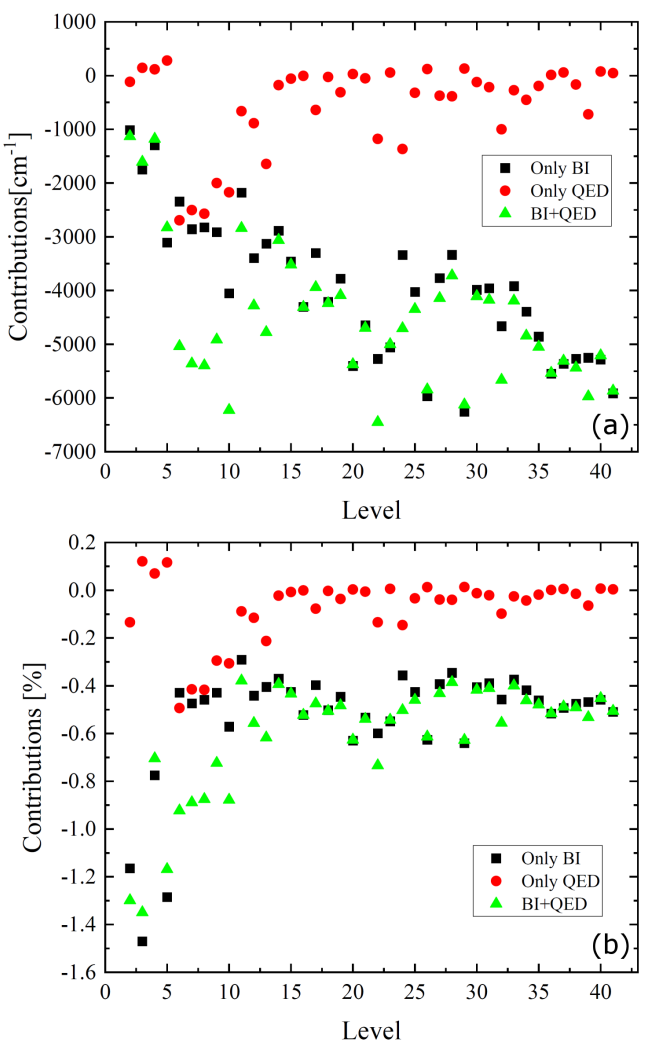

Fig. 4. The effects of the BI and QED corrections on the excitation energies of the Rb(XXIII) configurations obtained from the present MCDHF calculations in $\left[\mathrm{cm}^{-1}\right]$ (a) and percentage (b).

configurations have to be added to adequately represent electron correlations (i.e., mixing coefficients). These extra configurations are represented by CSFs and must have the same angular momentum and parity as the occupied orbitals [18]. For instance, the $3 s^{2} 3 p^{3}{ }^{4} S_{3 / 2}$ level is represented by $0.983 s^{2} 3 p^{3}{ }^{4} S_{3 / 2}$ and $0.093 s^{2} 3 p^{3}{ }^{2} P_{3 / 2}$. The former two mixing coefficients for the wave functions of the calculated levels are shown in Table II.
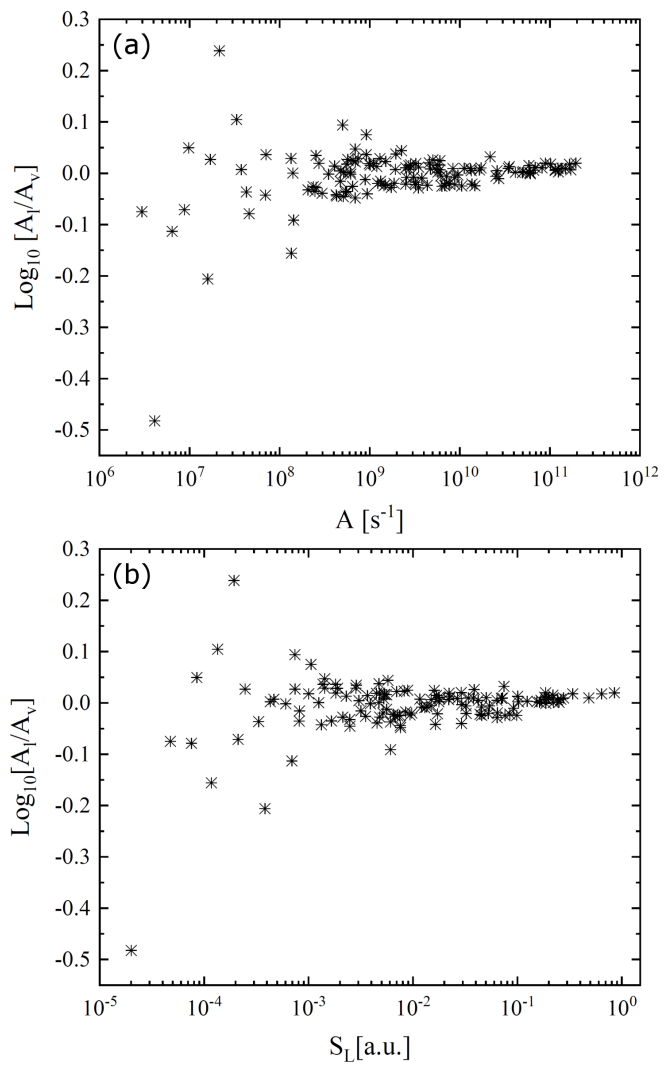

Fig. 5. A scatterplot of $\log \left(A_{l} / A_{v}\right)$ as a function of transition probability $A_{l}$ (a) and line strength $S_{l}$ (b) for the E1 transitions.

The most important contributions to the total wave function of a given level are those from the same configuration. For example, the configurationmixed wave function for the $3 s^{2} 3 p^{3}\left({ }_{3}^{4} S\right)^{4} S_{3 / 2}$ level is represented as $3 s^{2} 3 p^{3}{ }^{4} S_{3 / 2}=0.643 s^{2} 3 p^{3}{ }^{4} S_{3 / 2}+$ $0.243 s^{2} 3 p^{3}{ }^{2} P_{3 / 2}$, where 0.64 and 0.24 are contributions. The present and previous results are very close to one another in the description of the configuration-interaction wave functions. Because of the strong mixing, levels 12 and 15, 18 and 23, 28 and 38, 32 and 41 have the same quantum labels in the original output. With the help of JJ2LSJ [18], the levels mentioned above had been adjusted. In the present calculations, the nuclear parameters $I$, $\mu_{I}$, and $Q$ are all set to 1 . The $A_{J}$ and $B_{J}$ values for a specific isotope can be scaled with the tabulated values given in Table II.

Among the calculated wavelengths of transition between the lowest 41 levels in Rb(XXIII), the experimental results data compiled by NIST listed the observed wavelengths for seven E1 transitions. The observed results are from Sugar et al. [8], and the transition rates are from Huang [9]. The accuracy of the calculated $\mathrm{CV}$ and $\mathrm{VV}$ wavelengths relative to NIST can be assessed using Table III, where the agreement is within $0.10 \AA$ for CV. The difference between VV and NIST is in the range of $0.48-0.59 \AA$, while $\mathrm{CV}$ is in the 
TABLE II

The $L S$-composition, $A_{j}, B_{j}$ hyperfine interaction constants, and Landé $g_{J}$-factors for the lowest 41 levels in $\mathrm{Rb}(\mathrm{XXIII}), a(b)=a \times 10^{b}$.

\begin{tabular}{|c|c|c|c|c|c|c|}
\hline Key & Configuration & Mix & $L S$-composition & $A[\mathrm{MHz}]$ & $B[\mathrm{MHz}]$ & $g_{J}$ \\
\hline 1 & $3 s^{2} 3 p^{3}{ }^{4} S_{3 / 2}$ & $0.98(1)+0.09(5)$ & $0.64(1)+0.24(5)$ & $1.128(4)$ & $1.331(4)$ & 1.709 \\
\hline 2 & $3 s^{2} 3 p^{3}{ }^{2} D_{3 / 2}$ & $0.78(2)+0.51(1)$ & $0.61(2)+0.26(1)$ & $8.848(3)$ & $2.302(4)$ & 1.174 \\
\hline 3 & $3 s^{2} 3 p^{3}{ }^{2} D_{5 / 2}$ & $0.99(3)$ & $0.98(3)$ & $3.380(4)$ & $-9.019(1)$ & 1.197 \\
\hline 4 & $3 s^{2} 3 p^{3}{ }^{2} P_{1 / 2}$ & $0.98(4)$ & $0.97(4)$ & $9.583(4)$ & $0.000(0)$ & 0.663 \\
\hline 5 & $3 s^{2} 3 p^{3}{ }^{2} P_{3 / 2}$ & $0.79(5)+0.51(2)$ & $0.63(5)+0.26(2)$ & $1.921(4)$ & $-3.645(4)$ & 1.243 \\
\hline 6 & $3 s 3 p^{4}{ }^{4} P_{5 / 2}$ & $0.90(6)+0.29(25)$ & $0.81(6)+0.08(25)$ & $7.079(4)$ & $2.150(4)$ & 1.560 \\
\hline 7 & $3 s 3 p^{4}{ }^{4} P_{3 / 2}$ & $-0.85(7)-0.31(27)$ & $0.74(7)+0.10(27)$ & $3.699(4)$ & $-2.362(4)$ & 1.614 \\
\hline 8 & $3 s 3 p^{4}{ }^{4} P_{1 / 2}$ & $0.86(8)+0.37(13)$ & $0.74(8)+0.13(13)$ & $1.781(5)$ & $0.000(0)$ & 2.560 \\
\hline 9 & $3 s 3 p^{42} D_{3 / 2}$ & $0.68(9)-0.36(7)$ & $0.47(9)+0.13(7)$ & $6.231(3)$ & $-2.925(4)$ & 0.992 \\
\hline 10 & $3 s 3 p^{4^{2}} D_{5 / 2}$ & $-0.82(10)+0.42(35)$ & $0.67(10)+0.18(35)$ & $7.695(4)$ & $-5.209(4)$ & 1.230 \\
\hline 11 & $3 s^{2} 3 p^{2} 3 d\left({ }^{3} P\right)^{4} F_{3 / 2}$ & $0.66(11)-0.41(9)$ & $0.43(11)+0.17(9)$ & $6.008(3)$ & $2.341(3)$ & 0.751 \\
\hline 12 & $3 s^{2} 3 p^{2} 3 d\left({ }^{3} P\right)^{2} P_{3 / 2}$ & $0.55(12)-0.54(11)$ & $0.31(12)+0.30(11)$ & $1.015(4)$ & $-1.342(3)$ & 1.011 \\
\hline 13 & $3 s 3 p^{42} S_{1 / 2}$ & $0.50(13)+0.52(32)$ & $0.25(13)+0.26(32)$ & $6.577(4)$ & $0.000(0)$ & 1.150 \\
\hline 14 & $3 s^{2} 3 p^{2} 3 d\left({ }^{3} P\right)^{4} F_{5 / 2}$ & $0.75(14)+0.49(21)$ & $0.57(14)+0.24(21)$ & $5.463(3)$ & $1.410(4)$ & 1.129 \\
\hline 15 & $3 s^{2} 3 p^{2} 3 d\left({ }^{1} D\right)^{2} F_{5 / 2}$ & $-0.63(15)-0.52(41)$ & $0.39(15)+0.27(41)$ & $2.627(4)$ & $-1.101(4)$ & 0.941 \\
\hline 16 & $3 s^{2} 3 p^{2} 3 d\left({ }^{3} P\right)^{4} F_{7 / 2}$ & $0.91(16)+0.29(18)$ & $0.83(16)+0.08(18)$ & $9.589(3)$ & $8.659(3)$ & 1.232 \\
\hline 17 & $3 s^{2} 3 p^{2} 3 d\left({ }^{3} P\right)^{4} D_{1 / 2}$ & $-0.83(17)-0.40(13)$ & $0.69(17)+0.17(13)$ & $8.182(4)$ & $0.000(0)$ & 0.548 \\
\hline 18 & $3 s^{2} 3 p^{2} 3 d\left({ }^{3} P\right)^{4} D_{7 / 2}$ & $-0.64(18)+0.57(37)$ & $0.41(18)+0.33(37)$ & $1.239(4)$ & $2.220(4)$ & 1.243 \\
\hline 19 & $3 s^{2} 3 p^{2} 3 d\left({ }^{3} P\right)^{4} D_{3 / 2}$ & $0.85(19)-0.33(11)$ & $0.72(19)+0.11(11)$ & $1.169(4)$ & $-1.430(4)$ & 1.120 \\
\hline 20 & $3 s^{2} 3 p^{2} 3 d\left({ }^{3} P\right)^{4} F_{9 / 2}$ & $0.87(20)-0.47(22)$ & $0.76(20)+0.22(29)$ & $1.608(4)$ & $-4.559(3)$ & 1.281 \\
\hline 21 & $3 s^{2} 3 p^{2} 3 d\left({ }^{3} P\right)^{4} D_{5 / 2}$ & $0.70(21)-0.37(14)$ & $0.50(21)+0.14(14)$ & $1.662(4)$ & $-1.333(4)$ & 1.205 \\
\hline 22 & $3 s^{2} 3 p^{2} 3 d\left({ }^{3} P\right)^{2} P_{1 / 2}$ & $-0.52(22)-0.52(13)$ & $0.28(22)+0.26(13)$ & $1.244(5)$ & $0.000(0)$ & 1.067 \\
\hline 23 & $3 s^{2} 3 p^{2} 3 d\left({ }^{1} D\right)^{2} G_{7 / 2}$ & $-0.68(23)+0.52(18)$ & $0.46(23)+0.27(18)$ & $1.739(4)$ & $2.743(4)$ & 1.114 \\
\hline 24 & $3 s 3 p^{42} P_{3 / 2}$ & $0.65(24)+0.45(12)$ & $0.42(24)+0.19(12)$ & $-6.854(3)$ & $1.176(4)$ & 1.193 \\
\hline 25 & $3 s^{2} 3 p^{2} 3 d\left({ }^{3} P\right)^{4} P_{5 / 2}$ & $0.81(25)-0.31(21)$ & $0.66(25)+0.09(21)$ & $9.799(3)$ & $2.060(3)$ & 1.504 \\
\hline 26 & $3 s^{2} 3 p^{2} 3 d\left({ }^{1} D\right)^{2} F_{7 / 2}$ & $0.58(26)+0.59(23)$ & $0.33(26)+0.35(23)$ & $1.325(4)$ & $2.421(4)$ & 1.112 \\
\hline 27 & $3 s^{2} 3 p^{2} 3 d\left({ }^{3} P\right)^{4} P_{3 / 2}$ & $0.72(27)+0.49(38)$ & $0.52(27)+0.25(38)$ & $1.811(4)$ & $-7.991(3)$ & 1.513 \\
\hline 28 & $3 s^{2} 3 p^{2} 3 d\left({ }^{3} P\right)^{4} P_{1 / 2}$ & $-0.74(28)-0.39(33)$ & $0.55(28)+0.15(33)$ & $6.955(4)$ & $0.000(0)$ & 2.188 \\
\hline 29 & $3 s^{2} 3 p^{2} 3 d\left({ }^{1} D\right)^{2} G_{9 / 2}$ & $0.87(29)+0.47(20)$ & $0.76(29)+0.22(20)$ & $1.298(4)$ & $5.107(4)$ & 1.160 \\
\hline 30 & $3 s^{2} 3 p^{2} 3 d\left({ }^{1} S\right)^{2} D_{3 / 2}$ & $0.49(30)+0.59(40)$ & $0.23(30)+0.33(40)$ & $1.707(4)$ & $1.130(4)$ & 0.989 \\
\hline 31 & $3 s^{2} 3 p^{2} 3 d\left({ }^{3} P\right)^{2} D_{5 / 2}$ & $0.68(31)-0.44(35)$ & $0.47(31)+0.20(35)$ & $1.069(4)$ & $1.521(4)$ & 1.121 \\
\hline 32 & $3 s 3 p^{42} P_{1 / 2}$ & $-0.59(32)-0.44(22)$ & $0.35(32)+0.19(22)$ & $4.212(3)$ & $0.000(0)$ & 1.073 \\
\hline 33 & $3 s^{2} 3 p^{2} 3 d\left({ }^{1} D\right)^{2} P_{1 / 2}$ & $-0.77(33)-0.38(39)$ & $0.58(33)+0.15(39)$ & $-1.669(4)$ & $0.000(0)$ & 0.925 \\
\hline 34 & $3 s^{2} 3 p^{2} 3 d\left({ }^{1} D\right)^{2} D_{3 / 2}$ & $0.75(34)+0.38(9)$ & $0.57(34)+0.15(9)$ & $1.355(4)$ & $-9.406(3)$ & 0.978 \\
\hline 35 & $3 s^{2} 3 p^{2} 3 d\left({ }^{1} D\right)^{2} D_{5 / 2}$ & $-0.33(35)+0.50(36)$ & $0.11(35)+0.24(36)$ & $1.724(4)$ & $-5.017(3)$ & 1.106 \\
\hline 36 & $3 s^{2} 3 p^{2} 3 d\left({ }^{1} S\right)^{2} D_{5 / 2}$ & $0.63(36)+0.57(35)$ & $0.40(36)+0.32(35)$ & $1.095(4)$ & $3.925(4)$ & 1.192 \\
\hline 37 & $3 s^{2} 3 p^{2} 3 d\left({ }^{3} P\right)^{2} F_{7 / 2}$ & $0.78(37)-0.54(26)$ & $0.61(37)+0.30(26)$ & $1.632(4)$ & $-4.412(3)$ & 1.131 \\
\hline 38 & $3 s^{2} 3 p^{2} 3 d\left({ }^{1} D\right)^{2} P_{3 / 2}$ & $0.65(38)+0.48(12)$ & $0.42(38)+0.23(12)$ & $9.234(3)$ & $-8.137(3)$ & 1.225 \\
\hline 39 & $3 s^{2} 3 p^{2} 3 d\left({ }^{1} D\right)^{2} S_{1 / 2}$ & $0.70(39)+0.38(13)$ & $0.48(39)+0.15(13)$ & $9.066(4)$ & $0.000(0)$ & 1.804 \\
\hline 40 & $3 s^{2} 3 p^{2} 3 d\left({ }^{3} P\right)^{2} D_{3 / 2}$ & $-0.71(40)+0.35(30)$ & $0.51(40)+0.35(30)$ & $1.016(4)$ & $1.160(4)$ & 0.856 \\
\hline 41 & $3 s^{2} 3 p^{2} 3 d\left({ }^{3} P\right)^{2} F_{5 / 2}$ & $-0.44(41)+0.60(31)$ & $0.19(41)+0.36(31)$ & $1.102(4)$ & $-2.070(4)$ & 1.102 \\
\hline
\end{tabular}

range of 0.01-0.10 $\AA$. The transition rates from VV and $\mathrm{CV}$ are generally in good agreement except for the transition $3 s^{2} 3 p^{2} 3 d\left({ }^{1} D\right)^{2} D_{5 / 2}-3 s^{2} 3 p^{3}{ }^{2} D_{3 / 2}$ with a transition rate of $3.0 \times 10^{9} \mathrm{~s}^{-1}$ which deviates from the VV and CV results by about two orders of magnitude.
To check the accuracy of the present data for the E1 transitions between the length and velocity forms of transition probabilities, $\log \left(A_{l} / A_{v}\right)$ as a function of the length form of transition probability $A_{l}$ and as a function of line strength $S_{l}$ were shown in Fig. 5a and b, respectively. 
Spectral lines of $\mathrm{Rb}(\mathrm{XXIII}), a(b)=a \times 10^{b}$.

TABLE III

\begin{tabular}{c|c|c|c|c|c|c|c}
\hline \hline \multirow{2}{*}{ Upper level } & \multirow{2}{*}{ Lower level } & \multicolumn{3}{|c|}{ Wavelengths $[\AA]$} & \multicolumn{3}{c}{ Transition probabilities [s $\left.{ }^{-1}\right]$} \\
\cline { 3 - 8 } & & Exp. [8] & CV & VV & Exp. [9] & CV & VV \\
\hline $3 s^{2} 3 p^{2} 3 d\left({ }^{3} P\right)^{2} F_{7 / 2}$ & $3 s^{2} 3 p^{3}{ }^{2} D_{5 / 2}$ & 103.556 & 103.459 & 103.021 & $2.1(11)$ & $1.9(11)$ & $2.0(11)$ \\
$3 s^{2} 3 p^{2} 3 d\left({ }^{1} D\right)^{2} D_{5 / 2}$ & $3 s^{2} 3 p^{3}{ }^{2} D_{3 / 2}$ & 103.980 & 103.891 & 103.478 & $3.0(9)$ & $1.0(11)$ & $1.0(11)$ \\
$3 s^{2} 3 p^{2} 3 d\left({ }^{1} D\right)^{2} D_{3 / 2}$ & $3 s^{2} 3 p^{3}{ }^{2} D_{3 / 2}$ & 104.469 & 104.376 & 103.919 & $1.3(11)$ & $1.2(11)$ & $1.3(11)$ \\
$3 s^{2} 3 p^{2} 3 d\left({ }^{3} P\right)^{4} P_{3 / 2}$ & $3 s^{2} 3 p^{3}{ }^{4} S_{3 / 2}$ & 104.780 & 104.720 & 104.299 & $5.1(11)$ & $1.5(11)$ & $1.4(11)$ \\
$3 s^{2} 3 p^{2} 3 d\left({ }^{3} P\right)^{4} P_{5 / 2}$ & $3 s^{2} 3 p^{3}{ }^{4} S_{3 / 2}$ & 106.357 & 106.298 & 105.851 & $1.5(11)$ & $1.3(11)$ & $1.5(11)$ \\
$3 s^{2} 3 p^{2} 3 d\left({ }^{1} D\right)^{2} D_{5 / 2}$ & $3 s^{2} 3 p^{3}{ }^{2} D_{5 / 2}$ & 107.552 & 107.547 & 107.017 & $2.0(10)$ & $6.6(10)$ & $6.3(10)$ \\
$3 s^{2} 3 p^{2} 3 d\left({ }^{3} P\right)^{2} D_{5 / 2}$ & $3 s^{2} 3 p^{3}{ }^{2} P_{3 / 2}$ & 109.510 & 109.423 & 108.916 & $1.8(11)$ & $1.6(11)$ & $1.7(11)$
\end{tabular}

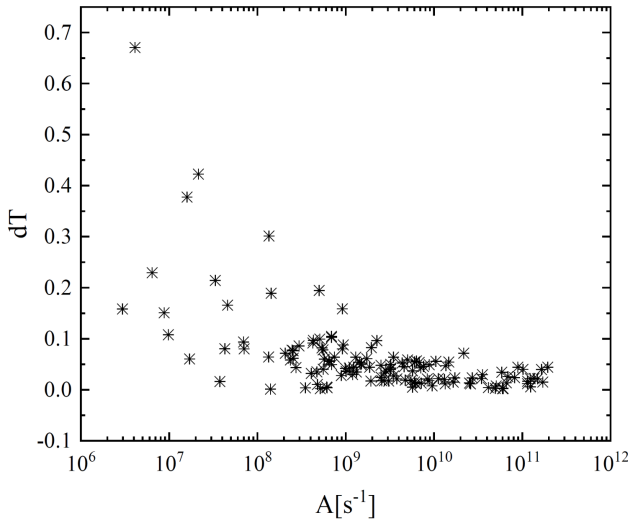

Fig. 6. A scatterplot of $\mathrm{d} T$ and $A\left[\mathrm{~s}^{-1}\right]$ for all E1 transitions.

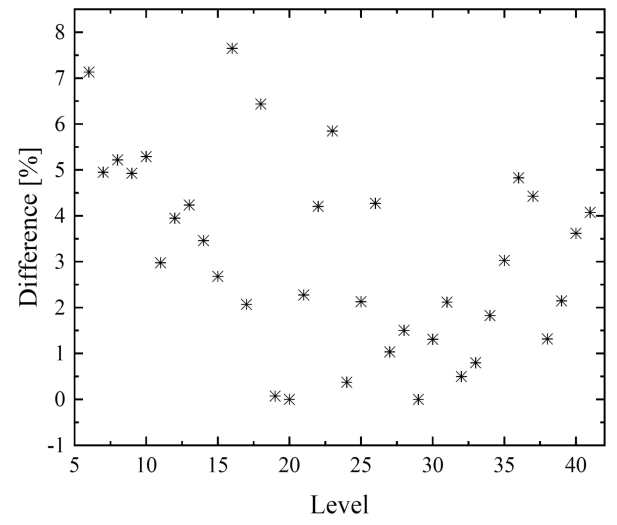

Fig. 7. A comparison of lifetime in length and velocity for $\mathrm{Rb}$ (XXIII).

The difference between $A_{l}$ and $A_{v}$ correlates with $A$-value much better than $S$-values, where the behavior of $\log \left(A_{l} / A_{v}\right)$ is regular with $A$-value (Fig. 5a), while the behavior with $S$-values is irregular (Fig. 5b).

Another check of the present data for the E1 transitions is the relative difference $(\mathrm{d} T)$ $\left(\mathrm{d} T=\operatorname{abs}\left(A_{l}-A_{v}\right) / \max \left(A_{l}, A_{v}\right) \quad\right.$ between the transition rates in length and velocity gauges. A value close to $\mathrm{d} T=0$ for an allowed transition is a known accuracy indicator [20]. In many cases, the values are reasonably close to 0 (see Fig. 6).
TABLE IV

The uncertainty percentage for A of the $\mathrm{E} 1$ transition in $\mathrm{Rb}$ (XXIII).

\begin{tabular}{c|c|l}
\hline \hline $\begin{array}{c}\text { Number of } \\
\text { transitions }\end{array}$ & Uncertainty $\%$ & \multicolumn{1}{|c}{ Uncertainty } \\
\hline 6 & 4.55 & $\mathrm{AAA}(\leq 0.3 \%)$ \\
8 & 6.06 & $\mathrm{AA}(\leq 1 \%)$ \\
20 & 15.15 & $\mathrm{~A}+(\leq 2 \%)$ \\
17 & 12.88 & $\mathrm{~A}(\leq 3 \%)$ \\
49 & 37.12 & $\mathrm{~B}+(\leq 7 \%)$ \\
17 & 12.88 & $\mathrm{~B}(\leq 10 \%)$ \\
7 & 5.30 & $\mathrm{C}+(\leq 18 \%)$ \\
4 & 3.03 & $\mathrm{C}(\leq 25 \%)$ \\
3 & 2.27 & $\mathrm{D}+(\leq 40 \%)$ \\
1 & 0.76 & $\mathrm{D}(\leq 50 \%)$ \\
\end{tabular}

But in other cases, for example, the difference of the $3 s^{2} 3 p^{2} 3 d\left({ }^{3} P\right)^{4} P_{3 / 2}-3 s^{2} 3 p^{3}{ }^{2} P_{3 / 2}$ transition can be larger than 0.6. According to the uncertain estimation suggested by Kramida [21], the following averaged uncertainty for $A$ values of E1 has been given in Table IV.

In particular, the presented calculations provide comprehensive new data for the E2, M1, and M2 transitions for $\mathrm{Rb}(\mathrm{XXIII})$, which no existent data for the public. This will help with the identification of spectral lines of $\mathrm{Rb}$ (XXIII). Owing to space limitations, full tables of the E1, E2, M1, and M2 transitions data will be provided by the authors on request.

The lifetime of the $j$ level is represented as $\tau=1 /\left(\sum_{j} A_{j i}\right)$. Lifetime is a measurable datum, and it can be a good check on the accuracy of this calculation. The uncertainty of the lifetime value is calculated using $\frac{\left|\tau_{l}-\tau_{v}\right|}{\max \left(\tau_{l}, \tau_{v}\right)}$ and plotted in Fig. 7 . The difference for all the excited levels considered here is up to $(\approx 3.13 \%)$. The present lifetime of the excited states from the CV $n=7$ calculation, compared with the results from the $n=7$ and $n=6$ results $\frac{\left|\tau_{n=7}-\tau_{6}\right|}{\max \left(\tau_{n=7}, \tau_{n=6}\right)}$ for the excited levels in $\mathrm{Rb}(\mathrm{XXIII})$, is presented in Fig. 8. The comparison shows good agreement between the two 


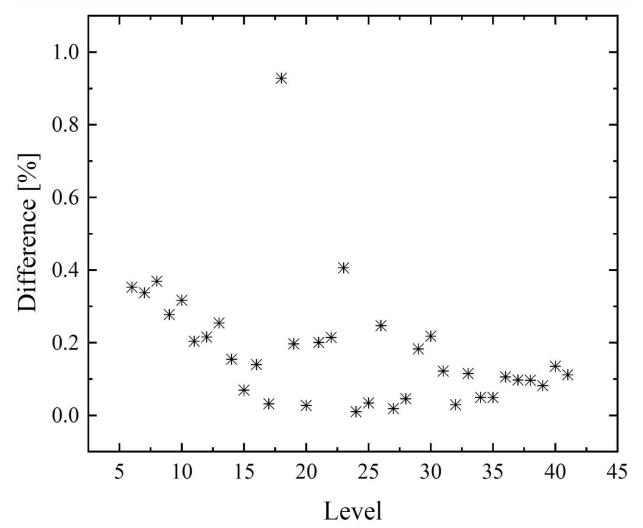

Fig. 8. A comparison of $\tau_{n=7}$ with $\tau_{n=6}$ for the excited levels in $\mathrm{Rb}$ (XXIII).

results, and the mean difference is within $0.30 \%$ except for the four excited levels, namely, $3 s 3 p^{4}{ }^{4} P_{5 / 2}$ (0.35\%), $3 s 3 p^{4}{ }^{4} P_{3 / 2}(0.34 \%), 3 s 3 p^{4}{ }^{4} P_{1 / 2}(0.37 \%)$, and $3 s 3 p^{4}{ }^{2} D_{5 / 2}(0.32 \%)$.

\section{Conclusion}

Energy levels, oscillator strengths, and transition probabilities for E1, E2, M1, and M2 transitions are presented for the lowest 41 levels of $\mathrm{Rb}$ (XXIII) belonging to the $3 s^{2} 3 p^{3}, 3 s 3 p^{4}$, and $3 s^{2} 3 p^{2} 3 d$ configurations. The valence-valence and core-valence correlation effects are accounted for systematically. The calculated energy levels and weighted oscillator strengths with the core-valence correlation effect show a good agreement with both theoretical and experimental data from the literature. The wavelengths are computed with almost spectroscopic accuracy, aiding line identification in spectra. Our results are useful for many applications such as astrophysics and atomic physics.

\section{Acknowledgments}

This work was supported by the Fundamental Research Funds for the Central Universities, Southwest Minzu University (grant No. 2021PTJS24), the Sichuan Department of Science and Technology (grant No. 2018JY0338), and the Key Natural Science Foundation of the Jiangsu Higher Education Institutions of China (grant No. 20KJA430005).

\section{References}

[1] S.A. Korotin, Astron. Lett. 46, 541 (2020).

[2] M.P. Roriz, M. Lugaro, C.B. Pereira, N.A. Drake, S. Junqueira, C. Sneden, Month. Notic. R. Astron. Soc. 501, 5834 (2021).
[3] C. Glaser, F. Karlewski, J. Kluge, J. Grimmel, M. Kaiser, A. Günther, H. Hattermann, M. Krutzik, J. Fortágh, Phys. Rev. A 102, 012804 (2020).

[4] A. Zelenski, G. Atoian, D. Raparia, J. Ritter, D. Steski, Rev. Sci. Instrum. 87, 02B705 (2016).

[5] J.E. Sansonetti, J. Phys. Chem. Ref. Data 35, 301 (2006).

[6] A. Kramida, J.R. Fuhr, Atomic Transition Probability Bibliographic Database, National Institute of Standards and Technology, Gaithersburg (MD) 2020.

[7] J. Sugar, V. Kaufman, J. Opt. Soc. Am. $B$ 1, 218 (1984).

[8] J. Sugar, V. Kaufman, W.L. Rowan, J. Opt. Soc. Am. B 8, 22 (1991).

[9] K.N. Huang, At. Data Nucl. Data Tables 30, 313 (1984).

[10] E. Träbert, Atoms 2, 15 (2014).

[11] P. Jönsson, G. Gaigalas, J. Bieroń, C. Froese Fischer, I.P. Grant, Comput. Phys. Commun. 184, 2197 (2013).

[12] C. Froese Fischer, G. Gaigalas, P. Jönsson, J. Bieroń, Comput. Phys. Commun. 237, 184 (2019).

[13] M.F. Gu, Can. J. Phys. 86, 675 (2008).

[14] F. Hu, J.M. Yang, C.K. Wang, L.F. Jing, S.B. Chen, G. Jiang, H. Liu, L.H. Hao, Phys. Rev. A 84, 042506 (2011).

[15] F. Hu, Y. Sun, M.F. Mei, C.C. Sang, J. Appl. Spectrosc. 85, 749 (2018).

[16] I.P. Grant, Relativistic Quantum Theory of Atoms and Molecules, Springer, New York 2007.

[17] B.J. McKenzie, I.P. Grant, P.H. Norrington, Comput. Phys. Commun. 21, 233 (1980).

[18] G. Gaigalas, C. Froese Fischer, P. Rynkun, P. Jönsson, Atoms 5, 6 (2017).

[19] J. Gillaspy, Trapping Highly Charged Ions: Fundamentals and Applications, Nova Publ., Hauppauge (NY) 2001.

[20] J. Ekman, M. Godefroid, H. Hartman, Atoms 2, 215 (2014).

[21] A. Kramida, Astrophys. J. Suppl. 212, 11 (2014). 